\title{
Validación de la Escala de Rendimiento Laboral Individual en Tra- bajadores Argentinos
}

\author{
Validation of the Job Performance Scale in Argentinean Workers
}

\author{
Sebastián Gabini $*{ }^{1},{ }^{2},{ }^{4}$, Solana Salessi ${ }^{3},{ }^{4}$ \\ 1 - Facultad de Psicología y Relaciones Humanas, Universidad Abierta Interamericana, Rosario, Argentina. \\ 2 - Facultad de Medicina y Ciencias de la Salud, Universidad Abierta Interamericana, Rosario, Argentina. \\ 3 - Facultad de Derecho y Ciencias Sociales del Rosario, Pontificia Universidad Católica Argentina. \\ 4 - Consejo Nacional de Investigaciones Cientificas y Técnicas (CONICET).
}

\author{
Introducción \\ Método \\ Resultados \\ Discusión \\ Conclusión \\ Referencias
}

\section{Resumen}

El objetivo del presente estudio fue validar y analizar las propiedades psicométricas de la Escala de Rendimiento Laboral Individual de Koopmans et al. (2013). Se ejecutó un estudio piloto con 31 estudiantes de posgrado para analizar la equivalencia operacional de la escala. Se elaboró un estudio principal a efectos de analizar las propiedades psicométricas de la escala puesta a punto en el estudio piloto. En esta ocasión se trabajó sobre una nueva muestra seleccionada en forma no probabilística, la que quedó integrada por 434 trabajadores. Sobre los datos recabados se ejecutaron análisis factoriales (exploratorio y confirmatorio). Sendos análisis indicaron una estructura trifactorial con adecuada consistencia (rendimiento en la tarea: $\alpha=.76$, comportamientos contraproducentes: $\alpha=.76$, y rendimiento en el contexto: $\alpha=.72$ ), confiabilidad compuesta (CR mayores a .70) y validez convergente (AVE mayor a .50). La versión argentina del instrumento quedó conformada por 13 ítems, demostrando ser una medida confiable y parsimoniosa del rendimiento laboral percibido.

Palabras clave: laboral, propiedades psicométricas, evidencia de estructura interna

\begin{abstract}
The aim of this study was to validate and analyze psychometric properties of Koopmans et al. (2013) Individual Work Performance Scale. A pilot study with 31 postgraduate students was performed to assay the operational equivalence of the scale. A main study was carried out in order to analyze the psychometric properties of the scale adapted in the pilot study. This time, the non-probabilistic sample comprised 434 workers. Factor analyses (exploratory and confirmatory) were executed over the data obtained. Both of them indicated a trifactorial structure with adequate internal consistency (task performance: $\alpha=.76$, counterproductive behaviors: $\alpha=.76$, and contextual performance: $\alpha$ $=.72$ ), composite reliability (CR greater than .70) and convergent validity (AVE greater than .50). The Argentinean version of the instrument was conformed by 13 items and proved to be a reliable and parsimonious scale to measure perceived job performance.
\end{abstract}

Keywords: performance, psychometric properties, internal structure evidence

\footnotetext{
* Correspondencia a: Sebastián Gabini. Dirección: Tucumán 4075, Rosario (2000), Argentina. Teléfono: +54 (0341) 155807086. sebastian_gabini@live. com.ar 


\section{Introducción}

El rendimiento laboral (RL) es uno de los constructos que mayor atención ha recibido por parte de los estudiosos del comportamiento organizacional. Posiblemente, su popularidad se deba a que la competitividad y la productividad de las organizaciones se encuentran íntimamente ligadas al desempeño individual de sus miembros (Koopmans et al., 2014). De allí que identificar sus determinantes y consecuentes haya sido uno de los objetivos prioritarios para los investigadores.

Con respecto a las variables predictoras, el metaanálisis realizado por O’Boyle, Humphrey, Pollack, Hawver y Story (2010) mostró que la inteligencia emocional desempeña un rol importante como antecedente del RL, junto con los cinco grandes factores de personalidad. En tanto que Dalal, Baysinger, Brummel, y LeBreton (2012), resaltaron el impacto de la autoeficacia, la necesidad de logro y algunas variables relacionadas con el entorno laboral, tales como el apoyo organizacional, el sistema de recompensas y las percepciones de justicia. Siguiendo la propuesta de Aarabi, Subramaniam, Almintisir y Akeel (2013) de probar el potencial predictivo de otros factores que podrían influir sobre el RL, la literatura más reciente presenta un gran número de estudios que proponen nuevos antecedentes. De esta manera, por ejemplo, se ha encontrado que el empoderamiento psicológico (Chiang \& Hsieh, 2012), y el compromiso laboral (Gruman \& Saks, 2011; Rich, LePine, \& Crawford, 2010), explican porciones significativas del RL.

En lo que hace a las consecuencias, tanto Díaz Cabrera et al. (2014) como Aguinis, Joo y Gottfredson (2011) resaltan tres tipos de benefi- ciarios de una gestión de rendimiento bien diseñada y ejecutada. En primer lugar se encuentran los empleados, quienes pueden experimentar una autoestima elevada, comprender mejor los comportamientos y resultados que requiere su posición e identificar de mejor manera los caminos para maximizar sus fortalezas y minimizar sus debilidades. En segundo lugar, los directivos, quienes desarrollan una fuerza laboral más motivada para desempeñarse, ganan un mayor predicamento entre sus subordinados, hacen que sus empleados sean más competentes y disfrutan de la diferenciación entre un buen y un mal rendimiento y de una comunicación más clara con los empleados acerca de su rendimiento. Finalmente, las organizaciones en su conjunto, las que realizan acciones administrativas más apropiadas, formulan más claramente las metas organizacionales, reducen las conductas inapropiadas de los empleados, se ven favorecidas con una mejor protección ante las demandas laborales, facilitan el cambio organizacional, y cuentan con empleados más comprometidos.

En cuanto a la conceptualización del RL, si bien se reconocen los aportes pioneros de Murphy (1990) referentes a la precisión de los alcances del término, en la actualidad se asiste a un renovado interés por establecer la naturaleza del concepto y diferenciarlo de otros constructos similares, tales como la productividad y la eficacia (Koopmans et al., 2013). En lo que hace a la naturaleza del RL, la tendencia vigente lo concibe como un constructo multidimensional y lo define como aquellos comportamientos que son relevantes para las metas organizacionales y se encuentran bajo el control del individuo (Koopmans et al., 2014). Estas precisiones conceptuales han permitido diferenciar al RL de otros conceptos asociados. Al respecto, hoy existe consenso en considerar al RL como los comportamientos orientados a la tarea (y los que 
sobrepasan las exigencias del rol, o comportamientos extra-rol), mientras que la productividad y la eficacia son entendidas como consecuencias de tales conductas.

Con respecto a la medición del constructo, la falta de un marco teórico unívoco ha favorecido la proliferación de numerosas medidas para evaluar el RL. Se trata de instrumentos que abarcan un amplio rango comprendido entre medidas objetivas de la productividad hasta instrumentos subjetivos tendientes a evaluar la calidad y cantidad del trabajo. La revisión de la literatura especializada (Koopmans et al., 2014; LePine, Erez, \& Johnson, 2002) indica cerca de 80 cuestionarios para medir el rendimiento laboral general y más de 40 escalas para evaluar el rendimiento en contextos específicos. Sin embargo, casi la totalidad de los instrumentos desarrollados hasta el momento no miden todas las dimensiones del constructo de forma conjunta o las operacionalizan de manera diferente. A su vez, el uso de distintas escalas para medir las dimensiones del RL conlleva el inconveniente de ítems redundantes, lo que aumenta las correlaciones entre los ítems e impacta negativamente sobre la validez de contenido (Koopmans, Bernaards, Hildebrandt, de Vet, \& Van der Beeket, 2014).

Frente a este panorama, Koopmans et al. (2013) desarrollaron un instrumento genérico para evaluar el constructo. De acuerdo a la operacionalización realizada por los autores, el RL está integrado por las siguientes cuatro dimensiones: rendimiento en la tarea, rendimiento en el contexto, comportamientos contraproducentes y rendimiento adaptativo. La primera dimensión comprende las conductas inherentes a las tareas técnicas del puesto. El rendimiento en el contexto abarca aquellas actividades direccionadas a mantener el ambiente interpersonal y psicológico en donde se desenvuelve el núcleo técnico. Los comportamientos contraproducentes incluyen toda acción intencional por parte de un miembro de la organización contraria a los legítimos intereses de la misma. Finalmente, el rendimiento adaptativo se orienta al grado hasta el cual un individuo es capaz de adaptarse a los cambios relacionados al rol laboral o a su ambiente. El instrumento, identificado con el nombre de Escala de Rendimiento Laboral Individual (IWPQ, Koopmans et al., 2013), ha sido desarrollado para medir las cuatro dimensiones del constructo como reflejo de las conductas de los trabajadores más que de la efectividad de las mismas.

Para la elaboración de la escala, Koopmans y sus colaboradores (2013) reunieron 128 indicadores a partir de una exhaustiva revisión de la literatura específica y de entrevistas con expertos. Luego de algunas depuraciones posteriores, obtuvieron una versión prototípica de la escala integrada por 47 ítems que valoraban las cuatro dimensiones del RL. La ejecución posterior de una prueba piloto sobre una muestra de 1181 trabajadores de cuello blanco (directivos, arquitectos, científicos), de cuello azul (carpinteros, mecánicos, conductores) y de cuello rosa (peluqueros, enfermeros, profesores), indicó la pertinencia de retener sólo tres dimensiones del RL, ya que la faceta correspondiente a rendimiento adaptativo surgió como un aspecto del rendimiento en el contexto. La versión final del instrumento quedó integrada por 18 ítems con formato tipo Likert de 5 puntos que evalúan tres dimensiones del RL, generalizables a todos los sectores laborales. La dimensión rendimiento en la tarea ( 5 ítems; $\alpha=$ .79) incluye indicadores que miden planeamiento y organización del trabajo, orientación a resultados, priorización del trabajo y eficiencia laboral. La dimensión rendimiento en el contexto ( 8 ítems; $\alpha=.83$ ) contiene indicadores que evalúan 
iniciativa, tareas laborales desafiantes, actualización de conocimientos y habilidades laborales y soluciones creativas frente a nuevos problemas. Mientras que la dimensión comportamientos laborales contraproducentes ( 5 ítems; $\alpha=.89$ ) nuclea indicadores que miden excesiva negatividad y acciones que dañan a la organización.

Hasta la fecha no se dispone de una versión de la Escala de Rendimiento Laboral Individual (Koopmans et al., 2013) traducida, adaptada y validada para su empleo con poblaciones hispanoparlantes. Por lo que, en un intento por llenar este vacío empírico-instrumental, el presente estudio fue diseñado con el propósito de establecer la equivalencia funcional del mencionado cuestionario en la población argentina.

\section{Método}

Diseño

El presente trabajo se inscribe en la categoría de investigaciones instrumentales (Ato, López, \& Benavente, 2013) desde el momento en que se orienta a la adaptación, validación y análisis psicométrico de un instrumento de medición. El trabajo se estructura en base a dos estudios complementarios rotulados como Estudio piloto y Estudio principal, respectivamente. La ejecución de ambos estudios se llevó a cabo en un todo de acuerdo con los lineamientos éticos establecidos por la Asociación Americana de Psicología (2010) y las recomendaciones del CONICET para las investigaciones en las ciencias sociales y humanas (Resolución 2827/06).

Estudio piloto
El objetivo de este primer estudio fue traducir y adaptar para la población argentina la Escala de Rendimiento Laboral Individual (Koopmans et al., 2013). Con tal propósito, se determinó la validez de contenido, el grado de equivalencia semántica y el grado de equivalencia operacional entre la versión argentina y la versión inglesa del instrumento (Muñiz, Elosua, \& Hambleton, 2013).

En primer lugar, todos los ítems fueron sometidos a revisión crítica por parte de tres profesionales especializados en psicología del trabajo y las organizaciones. Los especialistas recibieron un cuadernillo que contenía la definición conceptual y operacional del constructo rendimiento laboral y de cada una de las dimensiones contempladas en el instrumento (rendimiento laboral en la tarea, rendimiento laboral en el contexto, y comportamientos contraproducentes), así como la totalidad de los ítems que integraban la escala. La consigna directriz fue que clasificaran, según su criterio experto, cada ítem en la dimensión correspondiente. Asimismo, fueron alentados a realizar comentarios y sugerencias tendientes a mejorar la versión prototípica del instrumento.

La equivalencia semántica consiste en la traducción de los ítems, conservando el significado entre los idiomas involucrados. Para determinar esta equivalencia se siguió un procedimiento iterativo de traducción y re-traducción, usualmente identificado en la literatura bajo el nombre de traducción inversa (por su nombre en inglés backward translation). Este análisis fue realizado en tres etapas y contó con la participación de dos traductores profesionales. En primer lugar, el instrumento fue traducido del inglés al español (argentino). Seguidamente, los expertos en lengua inglesa retradujeron la versión argentina al idio- 
ma original. Por último, los mismos traductores compararon a ciegas las dos versiones del instrumento a efectos de identificar la concordancia entre cada ítem y su traducción en función de cuatro niveles de equivalencia: inalterado, poco alterado, bastante alterado y completamente alterado.

La equivalencia operacional se refiere al mantenimiento de las características operativas en cuanto a la claridad de las instrucciones, la adecuación semántica y sintáctica de los ítems y el tiempo que demanda completar la tarea. Para el análisis de esta equivalencia se efectuó un estudio piloto sobre una muestra por disponibilidad de 31 estudiantes de postgrado (53\% varones; 34 años de edad promedio, $\mathrm{DE}=5.45$ ), quienes aceptaron voluntariamente responder el protocolo. Una vez concluida la aplicación del instrumento se habilitó un espacio de intercambio para que los participantes opinaran acerca de la claridad de los ítems, posibles ambigüedades, tiempo requerido para responderlos y aspectos similares.

\section{Estudio principal}

El objetivo de este estudio fue determinar las propiedades psicométricas de la adaptación argentina de la Escala de Rendimiento Laboral Individual de Koopmans et al. (2013). Con tal propósito, se efectuaron análisis factoriales de carácter exploratorio y confirmatorio, así como los correspondientes análisis de confiabilidad y validez. Para este estudio se empleó la muestra y el procedimiento que se describen a continuación.

\section{Participantes}

Se trabajó con una muestra no probabilística integrada por 434 trabajadores $(52.6 \%$ va- rones). El mayor porcentaje de los participantes tenía edades comprendidas entre los 21-30 años (47.4\%) y los 31-40 años (23.7\%). Porcentajes menores se ubicaron en los rangos etarios comprendidos entre los 41-50 (10.2\%), y mayores de 50 años (16.7\%); en tanto que el rango de los 18 20 años (1.9\%), estuvo escasamente representado. El $30.2 \%$ de los sujetos estaba casado, mientras que cerca de un $63 \%$ era soltero. La muestra incluyó empleados de organizaciones de diversos ramos de actividad tales como servicios (35.8\%), educación (24.2\%), comercio $(21.9 \%)$ e industria (18.1\%). En relación con la antigüedad laboral, el $46.5 \%$ de la muestra llevaba más de cinco años en su trabajo, el 39.5\% entre uno y cinco años, y el $14 \%$ se encontraba trabajando hacía menos de un año.

\section{Procedimiento de recolección de los datos}

Para la selección de la muestra se tomó contacto con diversas organizaciones públicas y privadas localizadas en la ciudad de Rosario y alrededores y se las invitó a participar del estudio. Con aquellas que aceptaron colaborar se pautaron días y horarios para concretar la recolección de los datos. Luego de explicar el propósito del estudio y asegurar el anonimato y la confidencialidad de los datos recabados, se procedió a trabajar sólo con los sujetos que aceptaron participar voluntariamente, luego de firmar una hoja de consentimiento informado.

\section{Instrumento}

La totalidad de la muestra respondió la Escala de Rendimiento Laboral Individual traducida al español y puesta a punto durante el Estudio 
piloto. La misma quedó conformada por 18 ítems con formato de respuesta tipo Likert de 5 puntos, que variaban entre $1=$ nunca y $5=$ siempre.

\section{Estrategia de análisis de datos}

En primer lugar, se obtuvieron los estadísticos descriptivos (medias y desviaciones estándar), los índices de asimetría y curtosis, y los índices de discriminación (a partir del cómputo de las correlaciones ítem-total corregidas) para cada uno de los ítems. Seguidamente, se obtuvieron los índices de adecuación muestral (pruebas de Kaiser-Meyer-Olkin y de esfericidad de Bartlett). La estructura subyacente a los ítems se determinó mediante un análisis factorial exploratorio (AFE), ejecutado sobre la mitad de la muestra. Atendiendo a la recomendación de contar con un mínimo de 200 observaciones (Lloret-Segura, Ferreres, Hernández, \& Tomás, 2014), se seleccionaron en forma aleatoria simple 217 casos de los 434 presentes en la matriz de datos. Por la naturaleza ordinal de los datos, se utilizó el método de los mínimos cuadrados no ponderados (ULS, por su nombre en inglés Unweighted Least Squares) basado en una matriz de correlaciones policóricas (Hoffmann, Stover, de la Iglesia, \& Liporace, 2013). Para determinar el número de factores se aplicó una estrategia analítica de dos pasos. Primero se ejecutó un análisis paralelo optimizado, extrayendo aleatoriamente $500 \mathrm{sub}$-matrices e implementando el análisis de rango mínimo y a continuación se efectuó la extracción de los factores sugeridos, optando por la rotación oblicua promin (Baglin, 2014). Complementariamente, se evaluó el scree test (tomando en cuenta los componentes ubicados por encima de la curva del gráfico de sedimentación). El criterio para la selección de los ítems fue que pesaran .40 o más sobre el factor y que no saturaran significativamente sobre más de un factor al mismo tiempo (Lloret-Segura et al., 2014). La confiabilidad preliminar del instrumento se evaluó mediante el estadístico alfa ordinal, diseñado específicamente para el tratamiento de variables categóricas (Gadermann, Guhn, \& Zumbo, 2012).

El modelo sugerido por el AFE fue verificado empíricamente mediante un análisis factorial confirmatorio (AFC), el que fue realizado sobre la mitad de la muestra restante (Lloret-Segura et al., 2014). El supuesto de multi-normalidad se verificó mediante el cálculo del coeficiente de Mardia normalizado (Bentler, 2006). Se empleó el método de estimación de máxima verosimilitud (ML, por su nombre en inglés Maximum Likelihood) con la corrección robusta de Satorra-Bentler (SB; Bentler, 2006; Satorra, 2002), recomendado cuando los datos provienen de escalas ordinales (Bentler, 2006). Para evaluar la bondad de ajuste de cada modelo se analizó que la corrección del $\mathrm{S}-\mathrm{B} \chi 2$ sobre los grados de libertad (S-B $\chi 2$ ) $g l$ ) fuera inferior a 3; que el índice de bondad de ajuste (GFI, Goodness of Fit Index) y el índice de ajuste comparativo (CFI, Comparative Fit Index) alcanzaran valores iguales o superiores a .90; y que el valor del error cuadrático medio de aproximación (RMSEA, Root Mean Square Error of Approximation) fuera inferior a .05. Asimismo, se examinó el Criterio de Información de Akaike (AIC, Akaike Information Criterion), a sabiendas de que cuanto menor sea su valor, más parsimonioso es el modelo (Hair, Black, Babin, Anderson, \& Tatham, 2010).

Sobre el modelo de medida sugerido por el AFC se ejecutaron análisis de confiabilidad y validez. La consistencia se determinó en base al cómputo del coeficiente de confiabilidad com- 
puesta (CR, Composite Reliability). La validez convergente, vale decir, la varianza común entre los indicadores y su constructo se verificó mediante el cálculo de la varianza media extraída (AVE, Average Variance Extracted). Valores superiores a .50 son considerados evidencia de adecuada validez convergente, habida cuenta de que indican que más del $50 \%$ de la varianza del constructo es debida a sus indicadores (Fornell \& Larcker, 1981). Para valorar la validez discriminante la literatura actual (Bagozzi \& Yi, 2012; Henseler, Ringle, \& Sarstedt, 2015) recomienda tomar como criterio la raíz cuadrada del AVE. Si el valor obtenido para cada variable latente es superior a la correlación entre ésta y las restantes variables del modelo se puede asumir que cada factor comparte más varianza con sus indicadores que con los demás. El procesamiento y análisis de datos se realizó con los programas SPSS (versión 16), Factor versión 9.2 (Lorenzo-Seva \& Ferrando, 2013), y EQS 6 (Bentler, 2006).

\section{Resultados \\ Estudio piloto}

Validez de contenido y equivalencia semántica y operacional. La revisión del contenido de cada ítem por parte de los profesionales indicó que los mismos cubrían los tres dominios del rendimiento laboral postulados por Koopmans et al. (2013). A su vez, los índices de concordancia inter-jueces calculados a partir de la clasificación de los ítems, fueron altamente satisfactorios, oscilando entre .86 y $.90(p<.001)$. En lo que hace a la terminología empleada en la redacción de los ítems, los especialistas señalaron que la misma reflejaba el vocabulario habitual de la población argentina, por lo que no se juzgaron necesarias modificaciones adicionales. Respecto a la adaptación lingüística, ambos profesionales indicaron que se había mantenido inalterada la concordancia entre cada uno de los ítems en inglés y sus respectivas traducciones al español, por lo que concluyeron que la versión argentina de la Escala de Rendimiento Laboral Individual presentaba una adecuada equivalencia semántica. En lo que hace a la equivalencia operacional, los participantes del estudio piloto señalaron que las instrucciones para realizar la tarea estaban claramente redactadas, que no tuvieron inconvenientes para comprender el contenido de los ítems y que la escala utilizada para responder los ítems no les generaba dificultades.

\section{Estudio principal}

Análisis preliminares. La Tabla 1 presenta los estadísticos descriptivos, índices de asimetría y curtosis e índices de discriminación para cada ítem. De los 18 ítems, 12 presentaron valores de asimetría y curtosis comprendidos entre $-1 \mathrm{y}+1$. Los seis ítems restantes presentaron índices inferiores a 1.60 , los que fueron evaluados como aceptables (Hair et al., 2010).

En lo que respecta a la capacidad discriminativa, todos los ítems mostraron correlaciones positivas.

Análisis factorial exploratorio. La matriz de datos fue considerada factorizable habida cuenta de que el test de esfericidad de Bartlett fue significativo $(\chi 2=1079,122 ; p<.001)$ y el test de adecuación muestral de Kaiser-Meyer-Olkin arrojó un valor de .81. El análisis paralelo optimizado sugirió tres factores con autovalores superiores a sus equivalentes de la matriz de datos aleatorios. El gráfico de sedimentación también indicó una solución de tres factores, tal como puede observarse en la Figura 1. 
Gabini \& Salessi, 2016 , 16(1), 31-45

38

Tabla 1

Estadísticos descriptivos, índices de asimetría y curtosis y correlación ítem-total corregida correspondientes a los ítems de la Escala de Rendimiento Laboral Individual (versión adaptada).

\begin{tabular}{cccccc}
\hline ÍTEM & Media & DE & Asimetría & Curtosis & $\boldsymbol{r}$ i-total \\
\hline 1 & 3.20 & 1.10 & .52 & .83 & .68 \\
2 & 3.48 & 1.05 & .64 & .51 & .72 \\
3 & 3.31 & 1.13 & .77 & .86 & .84 \\
4 & 3.43 & .89 & -1.51 & -1.44 & .61 \\
5 & 3.19 & 1.04 & -.78 & .58 & .84 \\
6 & 3.16 & 1.16 & -.89 & -.92 & .82 \\
7 & 3.22 & 1.11 & -.55 & .71 & .79 \\
8 & 3.26 & 1.17 & .71 & -.66 & .72 \\
9 & 3.30 & 1.14 & .44 & .52 & .59 \\
10 & 3.28 & 1.09 & -.34 & -.93 & .61 \\
11 & 3.36 & 1.15 & .42 & -.88 & .83 \\
12 & 3.16 & 1.02 & .50 & .68 & .74 \\
13 & 3.22 & 1.10 & .71 & .83 & .69 \\
14 & 3.29 & 1.15 & -.47 & -.23 & .71 \\
15 & 3.08 & .91 & -1.42 & 1.37 & .65 \\
16 & 3.20 & 1.15 & .57 & .38 & .80 \\
17 & 3.18 & 1.26 & -.72 & -.42 & .79 \\
18 & 3.32 & 1.17 & -.86 & -.56 & .70 \\
\hline
\end{tabular}

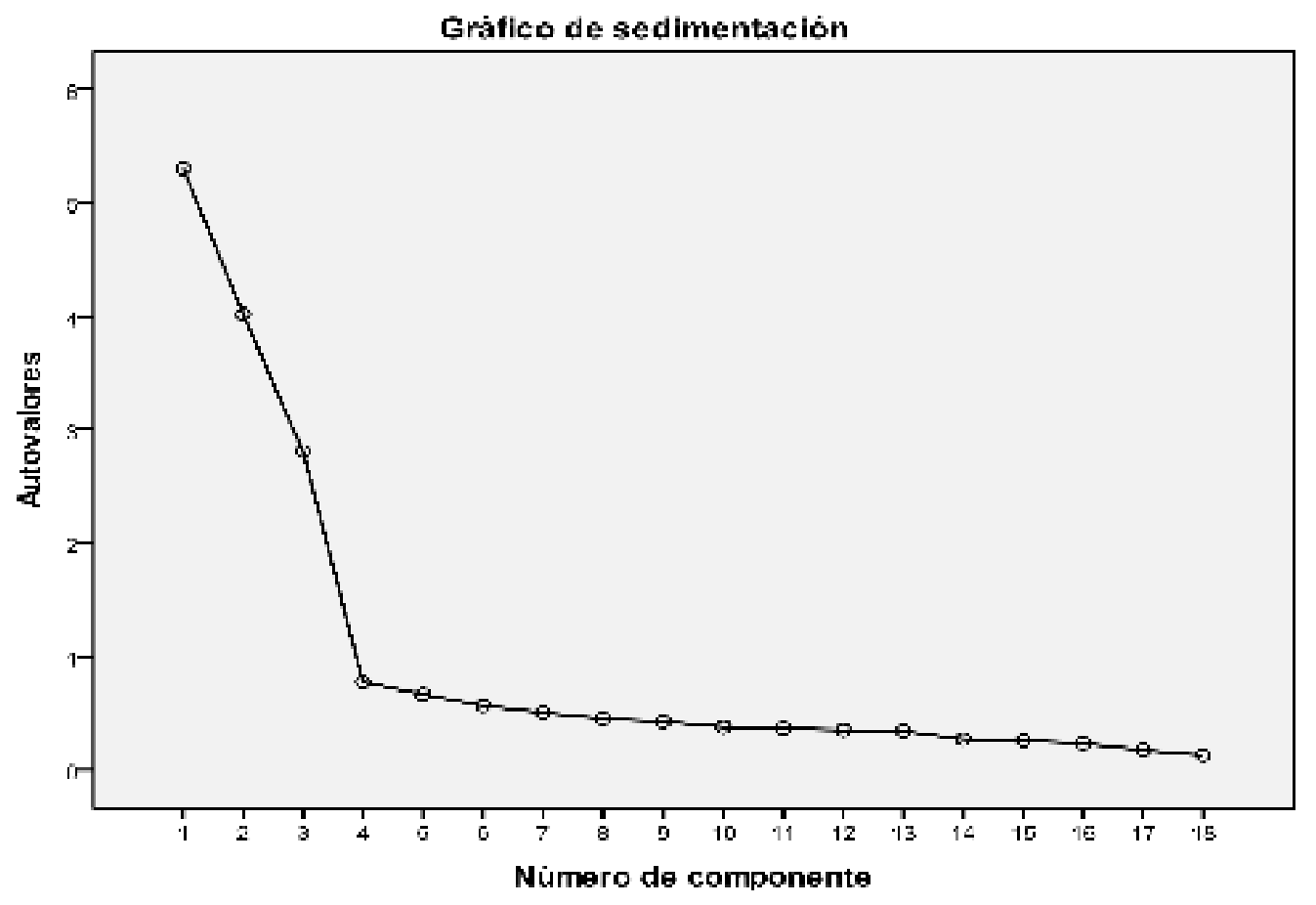


La inspección de las cargas factoriales condujo a eliminar dos ítems cuyas saturaciones no alcanzaron el umbral establecido (ítem 4: "asumí más responsabilidades laborales que las que me correspondian", e ítem 15: "en mi trabajo puedo separar las cuestiones principales de las secundarias"), de modo que se retuvieron 16 de los 18 reactivos de la escala original (Tabla 2). El cálculo de un segundo AFE, extrayendo los tres factores sugeridos inicialmente y aplicando la rotación promin ratificó dicha solución. El porcentaje global de varianza común explicada por los tres

\section{Tabla 2}

Matriz de configuración correspondiente a los ítems de la Escala de Rendimiento Laboral Individual (versión adaptada).

\section{CONTENIDO DEL ÍTEM}

Factor 1: Rendimiento en la tarea

En los últimos tres meses...

1. Fui capaz de hacer bien mi trabajo porque le dediqué el tiempo y el esfuerzo necesarios

2. Se me ocurrieron soluciones creativas frente a los nuevos problemas

3. Cuando pude realicé tareas laborales desafiantes

4. Cuando terminé con el trabajo asignado, comencé nuevas tareas sin que me lo pidieran

5. En mi trabajo, tuve en mente los resultados que debía lograr

6. Trabajé para mantener mis conocimientos laborales actualizados

7. Seguí buscando nuevos desafíos en mi trabajo

Factor 2: Comportamientos contraproducentes

En los últimos tres meses...

8. Me quejé de asuntos sin importancia en el trabajo

9. Comenté aspectos negativos de mi trabajo con mis compañeros

10. Agrandé los problemas que se presentaron en el trabajo

.70

11. Me concentré en los aspectos negativos del trabajo, en lugar de enfocarme en las cosas positivas

12. Comenté aspectos negativos de mi trabajo con gente que no pertenecía a la empresa

Factor 3: Rendimiento en el contexto

En los últimos tres meses...

13. Planifiqué mi trabajo de manera tal que pude hacerlo en tiempo y forma

14.Trabajé para mantener mis habilidades laborales actualizadas

15. Participé activamente de las reuniones laborales

.54

16. Mi planificación laboral fue óptima 
Análisis factorial confirmatorio. El valor obtenido en el Coeficiente de Normalidad Multivariada Normalizado se ubicó fuera del rango (entre -3 y 3) sugerido por Bentler (2006). Frente a este panorama se decidió utilizar estimadores robustos para la ejecución del AFC. Siguiendo los lineamientos de la estrategia de modelización confirmatoria, el modelo derivado del AFE, integrado por tres factores latentes correlacionados con 16 ítems como indicadores observables y sus respectivos errores de medición (Modelo A) se con- trastó empíricamente sobre la segunda mitad de la muestra (Lloret-Segura et al., 2014). Tal como puede observarse en la Tabla 3, las medidas de bondad de ajuste calculadas para este modelo no fueron totalmente satisfactorias.

Tabla 3

Índices de bondad de ajuste de los modelos examinados.

\begin{tabular}{lccccc}
\hline Modelos & S-B $\boldsymbol{\chi} \mathbf{2} / \boldsymbol{g} \boldsymbol{l}$ & GFI & CFI & RMSEA & AIC \\
\hline Modelo A (3 factores, 16 ítems) & 3.91 & .89 & .87 & .07 & 596.25 \\
Modelo B (3 factores, 13 ítems) & 2.40 & .95 & .97 & .04 & 436.14 \\
\hline
\end{tabular}

Frente a este panorama, se procedió a ajustar el modelo siguiendo las recomendaciones de los especialistas en el tema (Aguinis \& Edwards, 2014; Hair et al., 2010; Kline, 2011). Para ello se examinó la significación de las cargas factoriales, los índices de modificación y los residuos de la matriz de covarianzas. Con base en la información proporcionada por tales indicadores se decidió eliminar tres reactivos (ítem 3: "cuando pude realicé tareas laborales desafiantes", ítem 4: "cuando terminé con el trabajo asignado comencé nuevas tareas sin que me lo pidieran", e ítem 12: "comenté aspectos negativos de mi tra- bajo con gente que no pertenecía a la empresa"). El modelo re-especificado (Modelo B, Tabla 3), conformado ahora por tres factores correlacionados, 13 ítems como variables observables y sus respectivos errores de medición se sometió a un nuevo AFC. Los resultados obtenidos en esta oportunidad mostraron una significativa mejoría respecto del modelo anterior, demostrando que dicha estructura no era significativamente diferente de la indicada por la matriz de covarianza de los datos. El modelo de medida final correspondiente a la escala se muestra en la Figura 2. 


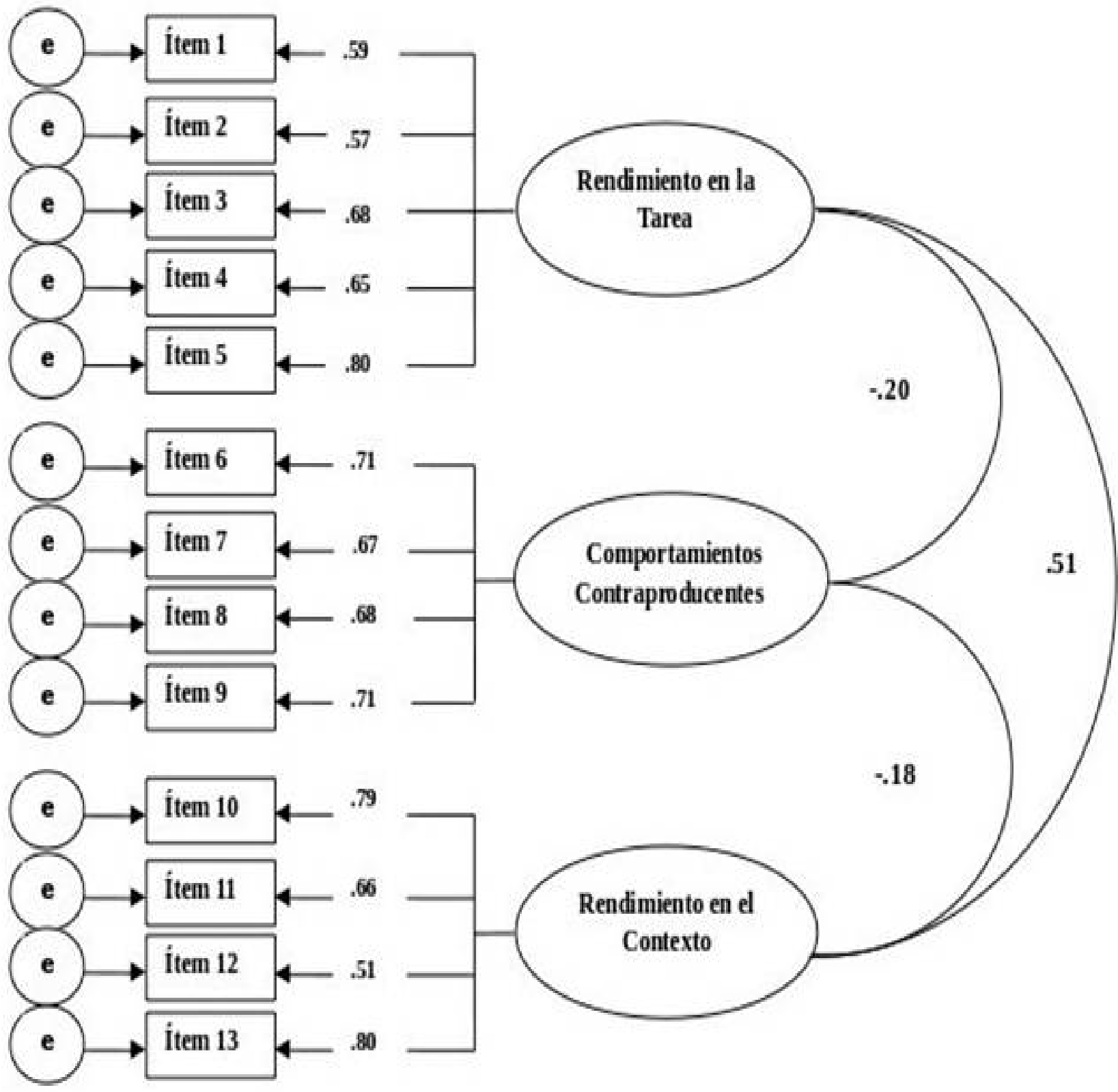

Figura 2

Modelo de medida correspondiente a la adaptación argentina de la Escala de Rendimiento Laboral Individual. drada superó la correlación entre los constructos.

La Tabla 4 presenta las covarianzas entre las tres facetas del rendimiento, los coeficientes $\mathrm{CR}$, el índice AVE y la raíz cuadrada del mismo. La inspección de la tabla precedente indica que cada sub-escala presenta adecuada confiabilidad. El coeficiente AVE se ubicó por encima del mínimo recomendado, y en todos los casos su raíz cuaTales resultados indican que la varianza capturada por los factores identificados es mayor que la debida a los errores de medida, y se puede explicar adecuadamente a través de los indicadores elegidos. 
Tabla 4

Covarianzas, medidas de confiabilidad y validez correspondientes a la Escala de Rendimiento Laboral Individual (versión adaptada).

\begin{tabular}{lccccc}
\hline & $\mathbf{1}$ & $\mathbf{2}$ & $\mathbf{3}$ & $\mathbf{C R}$ & AVE \\
\hline 1. Rendimiento en la tarea & $(.74)$ & -.20 & $\mathbf{. 5 1}$ & .74 & .55 \\
2. Comportamientos contraproducentes & & $(.73)$ & $\mathbf{- . 1 8}$ & .73 & .54 \\
3. Rendimiento en el contexto & & & $(.72)$ & .70 & .52 \\
\hline
\end{tabular}

\section{Discusión}

El objetivo del presente estudio fue validar la Escala de Rendimiento Laboral Individual de Koopmans et al. (2013). A partir del trabajo realizado, se ha logrado adaptar para su empleo con muestras hispanoparlantes un instrumento con adecuadas propiedades psicométricas para medir el rendimiento laboral, sin discriminar en función del tipo de actividad que realice el trabajador. En primer lugar, el AFE permitió identificar la existencia de un modelo oblicuo de tres factores, el que, con pequeños ajustes, fue confirmado mediante los AFC realizados. La estructura final obtenida indica que las dimensiones de rendimiento en la tarea, rendimiento en el contexto y comportamientos contraproducentes constituyen tres aspectos diferentes, aunque vinculados del rendimiento laboral. Esta conclusión es consistente con la estructura y los fundamentos teóricos de la escala original (Koopmans et al., 2013) al tiempo que se muestra en línea con los resultados informados por la validación inglesa del instrumento (Koopmans et al., 2016).

Por tratarse de un modelo con indicadores reflectivos (Aguinis \& Edwards, 2014), se asume que los ítems se encuentran correlacionados entre sí. Esto implica que pueden sustituirse unos por otros, vale decir, que son intercambiables. Por esa razón, el haber eliminado algunos ítems para conservar sólo los mejores indicadores no menoscaba en modo alguno la confiabilidad del instrumento aquí presentado. En efecto, tanto el coeficiente alfa ordinal como el coeficiente de confiabilidad compuesta obtenidos para cada sub-escala demuestran una adecuada homogeneidad y equivalencia de los ítems que integran la escala (Gadermann et al., 2012; Hair et al., 2010). Esta satisfactoria consistencia interna, permite concluir que es un instrumento confiable para la medición del rendimiento laboral. Asimismo, considerando que el coeficiente alfa para cada dimensión fue mayor que el correspondiente a la escala total, con finalidades prácticas podrían utilizarse las subescalas por separado, lo que transforma al instrumento en una herramienta versátil y parsimoniosa.

Los resultados obtenidos muestran, además, que la escala presenta una adecuada validez convergente desde el momento en que los ítems están significativa y fuertemente correlacionados con las variables latentes correspondientes (rendimiento en la tarea, comportamientos contraproducentes y rendimiento en el contexto), al tiempo que las cargas factoriales son elevadas, oscilando entre .51 y $.80(p<.001)$. En lo referente a la validez discriminante, los hallazgos señalan que cada factor de la escala comparte más varianza con sus indicadores observables que con los demás constructos del modelo. En conclusión, la escala se encuentra integrada por un conjunto de ítems que representan apropiadamente dimensiones distintas pero significativamente relacionadas del RL 
(Bagozzi \& Yi, 2012; Henseler et al., 2015).

\section{Limitaciones, fortalezas e implicancias prácticas del estudio}

Como toda investigación científica, el presente estudio no está exento de limitaciones. En primer lugar, es necesario efectuar algunas consideraciones en términos de la validez externa del estudio: la estabilidad de las dimensiones a lo largo del tiempo no ha sido probada, por lo que en estudios futuros sería recomendable que se investigue su confiabilidad test-retest. En segundo lugar, la representatividad de la muestra constituye otra posible limitación del estudio realizado, habida cuenta de que se ha trabajado con una muestra integrada por trabajadores provenientes de las zonas más industrializadas y desarrolladas del país. Por este motivo la generalización de los resultados requeriría estudios complementarios con un amplio rango de ocupaciones y trabajadores argentinos. Finalmente, cabe destacar que la escala aquí presentada evalúa el rendimiento laboral percibido, de modo que las respuestas podrían verse contaminadas por el componente subjetivo que esto conlleva, así como por tendencia de las personas a presentarse a sí mismas de un modo favorable y socialmente deseado. En relación con este último aspecto, cabe señalar que en el presente estudio se tomaron todos los recaudos necesarios para garantizar el carácter anónimo del protocolo de recolección de datos y la confidencialidad de la información. No obstante, sería importante que futuras investigaciones incluyeran una escala específica para medir esta tendencia.

Entre las principales fortalezas hay que destacar que la versión validada de la Escala de Rendimiento Laboral Individual resulta muy parsimoniosa y fácil de administrar. Es sabido que los instrumentos largos requieren más tiempo para ser completados, presentan mayores porcentajes de datos faltantes y suelen generar mayores tasas de rechazo (Schoorman \& Mayer, 2008). Por lo que disponer de un instrumento con adecuadas propiedades psicométricas e integrado por pocos ítems comporta ventajas prácticas para los estudiosos del comportamiento organizacional. En primer lugar, medidas objetivas de RL no son fácilmente obtenibles en todos los puestos de trabajo, quedando reservadas para aquellos que adoptan un sistema de gestión por objetivos. En segundo lugar, a menudo los empleados tienen más oportunidades que sus compañeros o supervisores para observar sus propios comportamientos; particularmente, cuando se trata de comportamientos contraproducentes. Finalmente, al tratarse de una escala genérica, es aplicable a una amplia diversidad de sectores ocupacionales, posibilitando además la comparación entre los mismos (Koopmans et al., 2013).

La medición de las percepciones de RL comporta implicancias prácticas para la organización. Por un lado, permite que los empleados asuman una posición más activa durante la evaluación, incentivándolos a explorar sus propias capacidades y limitaciones. Por otro lado, proporciona información valiosa susceptible de capitalizarse tanto en el diseño de estrategias de capacitación, como en el desarrollo de planes de carrera (Robbins \& Judge, 2009; Pablos-Teijeiro \& Biedma-Ferrer, 2013). En este sentido, mientras que las medidas objetivas posibilitan a la organización tener un panorama de los resultados alcanzados en relación con las metas proyectadas, las medidas subjetivas permiten explorar los comportamientos que cada empleado reconoce en sí mismo. De este modo, administradores y gerentes de recursos humanos pueden identificar a los empleados que cumplen o exceden lo esperado, al tiempo que determinar las competencias que los mismos deben desarrollar para maximizar su potencial. En última instancia, 
la información así obtenida podrá ser utilizada tanto para la toma de decisiones en torno a compensaciones, promociones y transferencias, como en el diseño de programas de capacitación y programación de la carrera profesional.

Como corolario de la investigación realizada, se puede concluir que el presente trabajo representa una contribución genuina para que otros estudios puedan ser llevados a cabo con el recurso de un instrumento con probadas propiedades psicométricas. La escala desarrollada puede, además, aportar información crucial para diagnósticos e intervenciones organizacionales. La validación de la Escala de Rendimiento Laboral Individual con muestras de sujetos argentinos deja abierta la necesidad de llevar a cabo futuros estudios de validación en otros contextos laborales y en otras culturas. Esa sería una vía idónea para verificar la validez transcultural del instrumento aquí presentado.

\section{Conclusión}

La presente investigación provee apoyo a la estructura trifactorial de la Escala de Rendimiento Laboral Individual y demuestra que el mismo es una medida válida y confiable. Estas notas distintivas transforman al instrumento validado en una herramienta útil para medir las percepciones en torno al propio rendimiento laboral al interior de las organizaciones argentinas.

\section{Referencias}

Aarabi, M. S., Subramaniam, I. D., Almintisir, A. B., \& Akeel, A. B. (2013). Relationship between motivational factors and job performance of employees in malaysian service industry. Asian Social Science, 9(9), 301-310. doi: 10.5539/ass. v9n9p301
Aguinis, H., \& Edwards, J. (2014). Methodological wishes for the next decade and how to make wishes come true. Journal of Management Studies, 51(1), 143-174. doi: 10.1111/ joms. 12058

Aguinis, H., Joo, H., \& Gottfredson, R. K. (2011). Why we hate performance management - And why we should love it. Business Horizons, 54(6), 503-507. doi: 10.1016/j.bushor.2011.06.001

American Psychological Association. (2010). Publication manual of the American Psychological Association (6 ${ }^{\mathrm{a}}$ ed.). Washington, DC: American Psychological Association.

Ato, M., López, J. J., \& Benavente, A. (2013). Un sistema de clasificación de los diseños de investigación en psicología. Anales de Psicología, 29(3), 1038-1059. doi: 10.6018/analesps.29.3.178511

Baglin, J. (2014). Improving your exploratory factor analysis for ordinal data: A demonstration using FACTOR. Practical Assessment, Research \& Evaluation, 19(5), 2-15. Recuperado de http://pareonline.net/getvn.asp?v=19\&n=5

Bagozzi, R., \& Yi, Y. (2012). Specification, evaluation, and interpretation of structural equation models. Journal of the Academy of Marketing Science, 40(1), 8-34. doi: 10.1007/ s11747-011-0278-x

Bentler, P. (2006). EQS 6 Structural Equations Program Manual. Encino, CA: Multivariate Software.

Chiang, C., \& Hsieh, T. (2012). The impacts of perceived organizational support and psychological empowerment on job performance: The mediating effects of organizational citizenship behavior. International Journal of Hospitality Management, 31(1), 180-190. doi: 10.1016/j. ijhm.2011.04.011

Consejo Nacional de Investigaciones Científicas y Técnicas, Comité de ética (2006). Lineamientos para el comportamiento ético en las Ciencias Sociales y Humanidades (Resolución No. 2857). Buenos Aires: Consejo Nacional de Investigaciones Científicas y Técnicas (CONICET).

Dalal, R. S., Baysinger, M., Brummel, B. J., \& LeBreton, J. M. (2012). The relative importance of employee engagement, other job attitudes, and trait affect as predictors of job performance. Journal of Applied Social Psychology, 42(1), 295-325. doi: 10.1111/j.1559-1816.2012.01017.x

Díaz-Cabrera, D., Hernández-Fernaud, E., Isla-Díaz, R., Delgado-Rodríguez, N., Díaz-Vilela, L., \& Rosales-Sánchez, C. (2014). Factores relevantes para aumentar la precisión, la viabilidad y el éxito de los sistemas de eva- 
luación del desempeño laboral. Papeles del Psicólogo, 35(2), 115-121. Recuperado de http://www.redalyc.org/ pdf/778/77831095004.pdf

Fornell, C., \& Larcker, D. F. (1981). Evaluating structural equations models with unobservable variables and measurement error. Journal of Marketing Research, 18(1), 39-50. doi: $10.2307 / 3151312$

Gadermann, A. M., Guhn, M., \& Zumbo, D. (2012). Estimating ordinal reliability for Likert-tipe and ordinal item response data: A conceptual, empirical, and practical guide. Practical Assessment, Research \& Evaluation, 17(3), 1-13. Recuperado de http://pareonline.net/pdf/v17n3.pdf

Gruman, J. A., \& Saks, A. M. (2011). Performance management and employee engagement. Human Resource Management Review, 21(2), 123-136. doi: 10.1016/j.hrmr.2010.09.004

Hair, J. E., Black, W. C., Babin, B. J., Anderson, R. E., \& Tatham R. L. (2010). Multivariate Data Analysis (6 $6^{\mathrm{a}}$ ed.). Upper Saddle River, NJ: Pearson-Prentice Hall.

Henseler, J., Ringle, C. M., \& Sarstedt, M. (2015). A new criterion for assessing discriminant validity in variance-based structural equation modelling. Journal of the Academy of Marketing Science, 43(1), 115-135. doi: 10.1007/s11747014-0403-8

Hoffmann, A., Stover, J., de la Iglesia, G., \& Fernández-Liporace, M. (2013). Correlaciones policóricas y tetracóricas en estudios factoriales exploratorios y confirmatorios. Ciencias Psicológicas, 7(2), 151-164. Recuperado de http:// www.scielo.edu.uy/scielo.php?script=sci_arttext\&pi$\mathrm{d}=$ S1688-42212013000200005

Kline, R. B. (2011). Principles and practice of structural equation modeling ( $3^{\mathrm{a}}$ ed.). New York, NY: Guilford.

Koopmans, L., Bernaards, C. M., Hildebrandt, V. H., Van Buuren, S., Van der Beek, A. J., \& De Vet, H. C. (2013). Development of an individual work performance questionnaire. International Journal of Productivity and Performance Management, 62(1), 6-28. doi: 10.1108/17410401311285273

Koopmans, L., Bernaards, C. M., Hildebrandt, V. H., De Vet, H. C., \& Van der Beek, A. J. (2014). Measuring individual work performance: Identifying and selecting indicators. Work, 48(2), 229-238.

Koopmans, L., Bernaards, C. M., Hildebrandt, V. H., Lerner, D., De Vet, H. C., \& Van der Beek, A. J. (2016). Cross-cultural adaptation of the Individual Work Performance Questionnaire. Work, 53(3), 609-619.
LePine, J. A., Erez, A., \& Johnson, D. E. (2002). The nature and dimensionality of organizational citizenship behavior: A critical review and meta-analysis. Journal of Applied Psychology, 87(1), 52-65.doi: 10.1037/0021-9010.87.1.52

Lloret-Segura, S., Ferreres, A., Hernández, A., \& Tomás, I. (2014). El análisis factorial exploratorio de los ítems: Una guía práctica, revisada y actualizada. Anales de Psicología, 30(3), 1151-1169. doi: 10.6018/analesps.30.3.199361

Lorenzo-Seva, U., \& Ferrando, P. J. (2013). FACTOR 9.2: A comprehensive program for fitting exploratory and semiconfirmatory factor analysis and IRT models. Applied Psychological Measurement, 37(6), 497-498. doi: 10.1177/0146621613487794

Muñiz, J., Elosua, P., \& Hambleton, R. (2013). Directrices para la traducción y adaptación de los tests: Segunda edición. Psicothema, 25, 151-157. Recuperado de http://www.psicothema.com

Murphy, K. R. (1990). Job performance and productivity. En K. R. Murphy \& F. E. Saal (Eds). Psychology in Organizations: Integrating science and practice (pp. 157-176). Hillsadle, NJ: Erlbaum.

O’Boyle, E. H., Humphrey, R. H., Pollack, J. M., Hawver, T. H., \& Story, P. A. (2011/2010). The relation between emotional intelligence and job performance: A meta-analysis. Journal of Organizational Behavior, 32(5), 788-818. doi: 10.1002/job.714

Pablos-Teijeiro, J. M., \& Biedma-Ferrer, J. M. (2013). La evaluación del rendimiento individual. Un instrumento válido para lograr la eficiencia en la gestión de recursos humanos en las administraciones públicas. Gestión y Análisis de Políticas Públicas, 10, 1-18. doi: 10.24965/gapp.v0i10.10115

Rich, B. L., LePine, J. A., \& Crawford, E. R. (2010). Job engagement: Antecedents and effects on job performance. Academy of Management Journal, 53(3), 617-635. doi: 10.5465/amj.2010.51468988

Robbins, S., \& Judge, T. (2009). Comportamiento organizacional (13 ${ }^{\mathrm{a}}$ ed.). México, DF: Pearson Educación.

Satorra, A. (2002). Asymptotic robustness in multiple group linear-latent variable models. Econometric Theory, 18(2), 297-312. doi: 10.1017.S0266466602182041

Schoorman, D. F. \& Mayer, R. C. (2008). The value of common perspectives in self-reported appraisals: You get what you ask for. Organizational Research Methods, 11, 148-159. doi: $10.1177 / 1094428107307168$ 